\title{
Interaction of alcohol with phospholipid membrane: NMR and XRD investigations on DPPC-hexanol system
}

\author{
U. Wanderlingh ${ }^{\text {a,* }}$, G. D’Angelo a , V. Conti Nibali ${ }^{\text {a }, ~ C . ~ C r u p i ~}{ }^{\text {a }}$, S. Rifici ${ }^{\text {a }}$, \\ C. Corsaro ${ }^{a}$ and G. Sabatino ${ }^{b}$

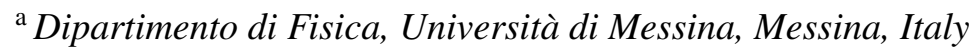 \\ ${ }^{\mathrm{b}}$ Dipartimento di Scienze della Terra, Università di Messina, Messina, Italy
}

\begin{abstract}
The investigations of the interaction between phospholipid bilayer and short-chain alcohols are relevant for the potential of lipid bilayer membranes to serve as model systems for studies of various biological processes including permeability of the plasma membrane and molecular mechanisms of anesthesia. Because the hydrophobic portion of an alcohol favorably interacts with lipid hydrocarbon chains, the polar hydroxyl group remains free to form hydrogen bonds with polar lipid atoms that are located near the water/lipid interface. Experiments on phospholipid membranes have shown that alcohols can induce an interdigitated phase and at high concentration even promote the assembly of some lipids into non-bilayer structures within the membrane interior. In this paper we have investigated the DPPC:hexanol system at high alcohol concentration (two molecules per phospholipid) by means of calorimetric, Nuclear Magnetic Resonance, X-ray diffraction and density measurements. We have found that the presence of a high alcohol concentration shifts the membrane transition temperature to lower values, and has a disordering effect on the phospholipid acyl chains in the gel phase. The bilayer spacing and the area of polar head have been also derived for the liquid phase.
\end{abstract}

Keywords: Biomolecules, phospholipid, alcohol, interdigitation, DSC, XRD, NMR

\section{Introduction}

Biological membranes play a crucial physical role in cells [8], they define the cell boundary and incorporate several proteins and functional compounds. The central core of any biological membrane is formed by amphiphilic molecules arranged side by side to form a lipid bilayer with head groups turned towards the surrounding water and apolar acyl chains in the membrane interior. Phospholipid bilayers exhibit lyotropic liquid crystalline properties and present a variety of phases as temperature is changed. From low to high temperatures [21], they pass from an highly ordered crystal/subgel phase $L_{c}$, to a gel phase $\left(L_{\beta}\right)$ which shows little disorder; then a fluid phase $\left(L_{\alpha}\right)$ is approached which shows a liquid-like disorder throughout the whole system (two-dimensional fluid). Just below $L_{\alpha}$, a ripple phase $\left(P_{\beta}\right)$ is found in which the lamellar structure is deformed by periodic modulations.

The interaction between lipid bilayer and small amphiphilic molecules, such as short-chained alcohols, presents interesting prospectives [18] concerning the potential of lipid bilayer membranes to serve as model systems for studies of various biological processes [3] including permeability of the plasma

\footnotetext{
* Corresponding author: U. Wanderlingh, Università di Messina, Sal. Sperone 31, I-98166 S Agata, Messina, Italy. Tel.: +39 0906 765023; E-mail: uwanderlingh@unime.it.
} 
membrane and molecular mechanisms of anesthesia. As a general picture of alcohol-membrane interactions it is assumed that small alcohols (up to three Carbon atom) mainly interact by competing with water for hydration sites on membrane surface, whereas alcohols with longer chain preferentially bind inserting their tail within the membrane. In both cases this results in the alteration of the compactness and the fluidity of the bilayer and in an increase of lateral pressure on transmembrane proteins $[2,4]$, by alcohols diffusing within the bilayer. These structural changes of the membrane can influence the conformation of proteins or other structures embedded in the membrane, providing a route in which alcohol or other small amphiphilic molecules influence the function of a cell. Experiments on phospholipid membranes have shown that alcohol can, for example, induce the interdigitated phase $L_{\beta I}$ and, at high concentrations, even promote the assembly of some lipids into non-bilayer structures within the membrane interior [1,6,7]. In an ordinary membrane the hydrophobic thickness is approximately twice the length of the hydrophobic tails of a phospholipid, in the interdigitated phase the hydrophobic thickness is reduced to the sum of the length of the hydrophobic tails of a single phospholipid and the alcohol. In such a case the normal bilayer structure changes into a monolayer in which two alcohol molecules screen the phospholipid tails from the water phase.

This kind of structure can be understood if we consider the location of the alcohol molecules in the bilayer. Due to the amphiphilic nature, the hydrophobic portion of the alcohol favorably interacts with lipid hydrocarbon chains, and the polar hydroxyl group remains free to form hydrogen bonds with polar atoms of the lipid head located near the water/lipid interface. So, lateral space is created between the head groups, leading to voids in the hydrophobic core. These voids are energetically unfavourable and thus the system will minimize the energy by the formation of an interdigitated phase $[5,13,17]$. There is also an entropic gain by replacing the highly ordered water molecules at the interface with alcohol molecules $[10,16,17]$. This location in the head group region disturbs the natural microstructure of the lipid membrane and is apparently responsible for the observed increases in membrane fluidity, permeability, lipid lateral mobility, for the decreasing main phase transition temperature and for the formation of an interdigitated phase.

In this paper we have investigated the DPPC:hexanol system at high alcohol concentration (two molecules per phospholipid) by means of calorimetric, Nuclear Magnetic Resonance, X-ray diffraction and density measurements. We were able to characterize the system phases and to obtain information on membrane structure and alcohol location.

\section{Methods and materials}

Differential scanning calorimetry (DSC) measurements were carried out by a Perkin-Elmer Pyris 1 . Samples were placed in a sealed aluminium pan to prevent dehydration during the thermal analysis. Thermograms were recorded at a rate of $1 \mathrm{~K} / \mathrm{min}$. To ensure thermal equilibrium was reached and to verify the occurrence of hysteresis, different scans at increasing and decreasing temperature were carried out for each sample.

High Resolution Magic Angle Spinning (HR-MAS) were carried out using a Bruker AVANCE NMR spectrometer operating at $700 \mathrm{MHz}{ }^{1} \mathrm{H}$-resonance frequency. Samples were spun at $10 \mathrm{kHz}$. Proton experiments were carried out at the resonance frequency of ${ }^{1} \mathrm{H}$. Resonance peaks were well resolved, with a typical peak line width of only $10-20 \mathrm{~Hz}$.

$X$-Ray Diffraction (XRD) were carried out by a D8-ADVANCE Bruker diffractometer in the BraggBentano geometry, using the $\mathrm{Cu} K \alpha_{1}$ line $(\lambda=1.542 \AA)$. During the measurements the samples were 
hosted in a small sample chamber with $180^{\circ}$ cylindrical Kapton windows. In the chamber the temperature was controlled by a PT100 and humidity was adjusted by adding water solution into an open reservoir.

Volumetric measurements on lipid/alcohol bilayer mixtures were performed by the neutral flotation method [12,24]. A small amount $(10 \mathrm{mg})$ of liposomes was dispersed in approximately $1 \mathrm{~g}$ of a $\mathrm{D}_{2} \mathrm{O}-\mathrm{H}_{2} \mathrm{O}$ mixture and centrifuged. The density of the lipid was obtained by varying the $\mathrm{D}_{2} \mathrm{O}-\mathrm{H}_{2} \mathrm{O}$ ratio until the suspension neither sank nor floated. Measurements were performed at $25^{\circ}$, and $3500 \mathrm{rpm}$ on a laboratory centrifuge for $1-12 \mathrm{~h}$.

The phospholipid 1,2-palmitoyl-sn-glycero-3-phosphocholine (DPPC) was purchased from Avanti Polar Lipids in powder form and used without further purification, hexanol was purchased by Sigma Chem. Co.

Samples for HR-MAS NMR and DSC experiments were prepared by dissolving the DPPC and hexanol, at 1:2 molar ratio, in excess of 2:1 $\mathrm{CHCl}_{3} / \mathrm{CH}_{3} \mathrm{OH}$ solution. The solvent was removed under $\mathrm{N}_{2}$ flux and the lipid/alcohol mixture was then diluted with $\mathrm{D}_{2} \mathrm{O}$ to a $10 \%$ by weight concentration. The solution was vortexed in order to obtain multilamellar vesicle, and then sonicated and subjected to temperature cycling between -4 and $50^{\circ} \mathrm{C}$ for $6 \mathrm{~h}$ to form small unilamellar liposomes and to obtain homogeneity.

Samples for XRD were prepared by spreading the solution of unilamellar liposomes on Silicon wafer and were left to equilibrate at $96 \%$ relative humidity for several days, the final water content was determinated by weighting.

\section{Results and discussion}

The thermograms obtained for DPPC and DPPC:hexanol [1:2] are shown in the inset of Fig. 1. In DPPC both pre and main transition $T_{m}$ are clearly observed $[11,22]$ at $36^{\circ} \mathrm{C}$ and $42^{\circ} \mathrm{C}$. The addition of a high hexanol concentration results in a broadening and in a shifting to the lower temperature of $30^{\circ} \mathrm{C}$ of the main transition, whereas the pre transition appears to be suppressed. These results confirm the disordering effect of alcohol on the lipid hydrocarbon chains and are in agreement with literature data on DPPC [9], and the data on DMPC [19] at lower alcohol concentration.

HR-MAS spectra for DPPC and DPPC:hexanol [1:2] in both $L_{\alpha}$ and $L_{\beta}$ phase are shown in Fig. 2. By an analysis of the chemical shift of ${ }^{1} \mathrm{H}$, the presence of hexanol in the membrane is revealed by a peak at $3.55 \mathrm{ppm}$ assigned to the first $\mathrm{CH}_{2}$ in the alcohol chain while the signal from the remaining $\mathrm{CH}_{2}$ is merged with that of the phospholipid chains at $1.28 \mathrm{ppm}$. In the $L_{\alpha}$ phase the spectra are quite similar, a small modification is observed at $4.20 \mathrm{ppm}$ and around $2.43 \mathrm{ppm}$ that are related to resonance in the glycerol group while resonances from the lipid core are almost unperturbed. In the gel phase the main differences are observed in the lipid tail region, indicating that the alcohol induces a bigger disorder in the acyl chains, consistent with an interdigitated phase. This is in agreement with the result of Feller [5], who found that methyl groups of ethanol have strong cross-relaxation with chain signals, suggesting that the alcohol molecules are oriented with their methyl groups towards the hydrophobic bilayer core. In our case this effect is even stronger due to the longer chain of hexanol.

The XRD spectra, shown in Fig. 2, for DPPC/hexanol/ $\mathrm{D}_{2} \mathrm{O}$ [1:2:7] were collected in the gel and liquid phase, and were compared to that of DPPC/ $\mathrm{D}_{2} \mathrm{O}$ [1:7] in the gel phase. Several Bragg peaks from the lamellar structure of the multi bilayers are clearly observed, albeit in the case of DPPC/hexanol/ $\mathrm{D}_{2} \mathrm{O}$ gel phase a distribution of distances seems more appropriate. From these data the lamellar spacing $D$ is obtained and corresponds to $60.0 \AA$ for DPPC, $57.5 \AA$ and $47.0 \AA$ for DPPC-hexanol in the liquid 


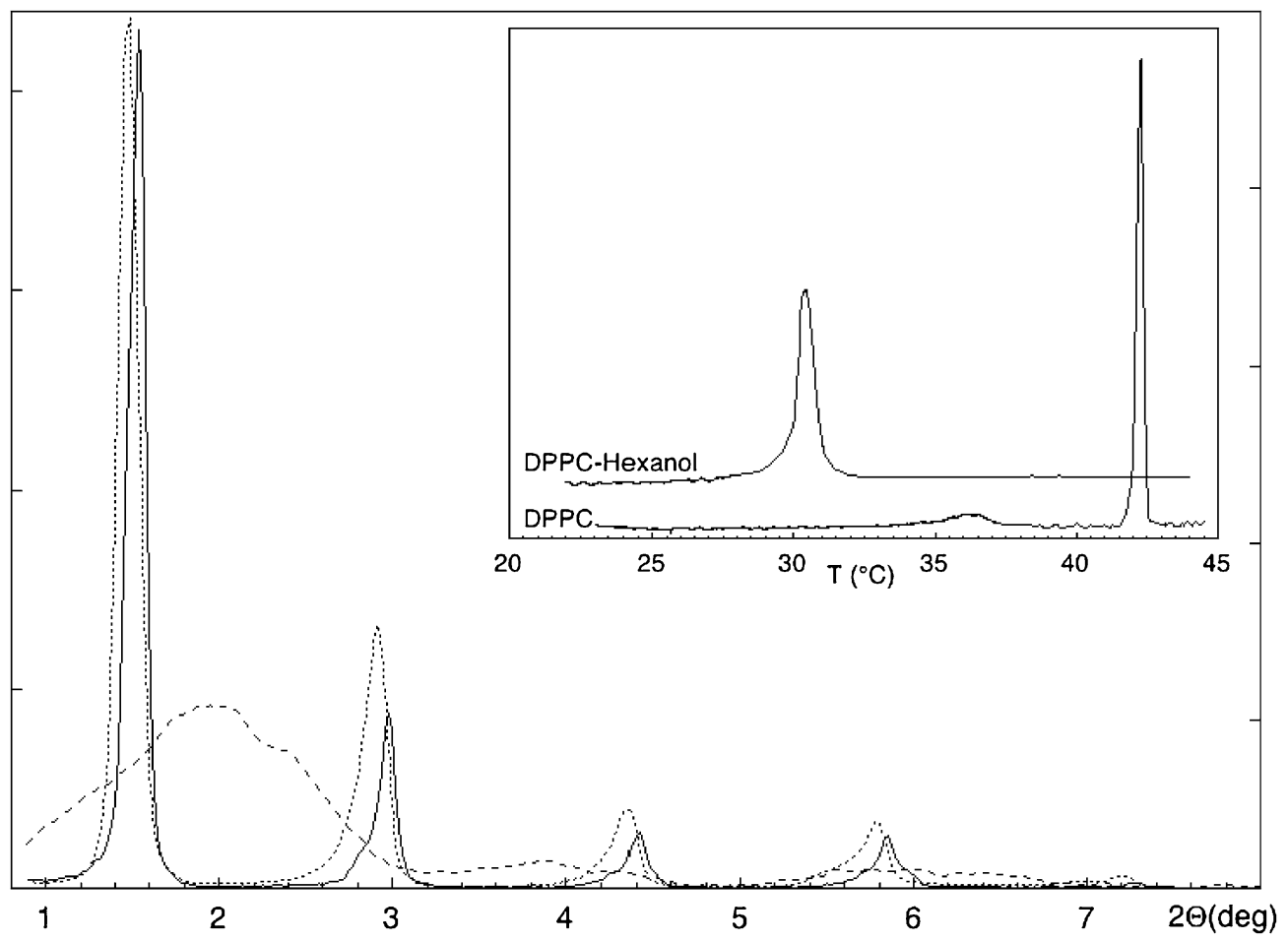

Fig. 1. X-ray diffraction spectra from DPPC gel (dotted line), DPPC:hexanol [1:2] in liquid (continuous line) and gel (dashed line) phases. The inset shows the thermograms of both systems.

and gel phase respectively. The shortening of $D$ in the gel phase of DPPC-hexanol accounts for an almost complete interdigitation, in fact the difference found in bilayer thickness amounts to $10.5 \AA$. Such a length corresponds to the difference in ten $-\mathrm{CH}_{2}-$ units between DPPC and hexanol acyl chains; assuming a length of $\simeq 1.0 \AA$ for chain unit, on the basis of a molecular model of alkane $[1,14]$. From the found lamellar spacing $D$ values and the molecular volumes, calculated by neutral flotation method, it is possible to determine the polar head area, $A$, for the investigated systems. In fact we can write for the volume of bilayer constituents (lipid and water) the relation: $\left(V_{1}+n_{\mathrm{w}} V_{\mathrm{w}}\right)=\mathrm{AD} / 2$, where $V_{1}$ is the phospholipid or phospholipid/alcohol volume, $n_{\mathrm{w}}$ is the number of hydration water molecules and $V_{\mathrm{w}}$ is the volume of a single water molecule, this latter estimated to $30 \AA^{3}$. That procedure was applied to DPPC gel and DPPC:hexanol liquid phase where the interlamellar distance is clearly identified, the results are shown in the Table 1. The obtained area of DPPC polar head in gel phase is in good agreement with literature [20,23] confirming the validity of the method. The increased value of $A$ in presence of hexanol $\left(+14 \AA^{2}\right)$ supports the location of alcohol head next to membrane polar head [7]. In fact the hexanol head area can be estimated as $6 \pm 1 \AA^{2}$, from accessible surface reconstruction of 1-hexanol.pdb. Moreover, we observe also a compacting of the alcohol within the bilayer. The hexanol neat volume is $208.5 \AA^{3}$ while the value found in DPPC is $189 \AA^{3}(-9.3 \%)$. This effect was also reported [15] in Molecular Dynamics simulation of hexanol in DMPC membrane, although to a lesser extent: $-7.4 \%$.

Concluding remarks. We have investigated the interaction of bio-mimetic membrane with hexanol by means of Calorimetric, Nuclear Magnetic Resonance, X-ray diffraction and accurate density measurements. To our knowledge this is the first study that combine this different techniques to obtain detailed structural information on membrane-alcohol systems. The obtained results support the evidence of an 


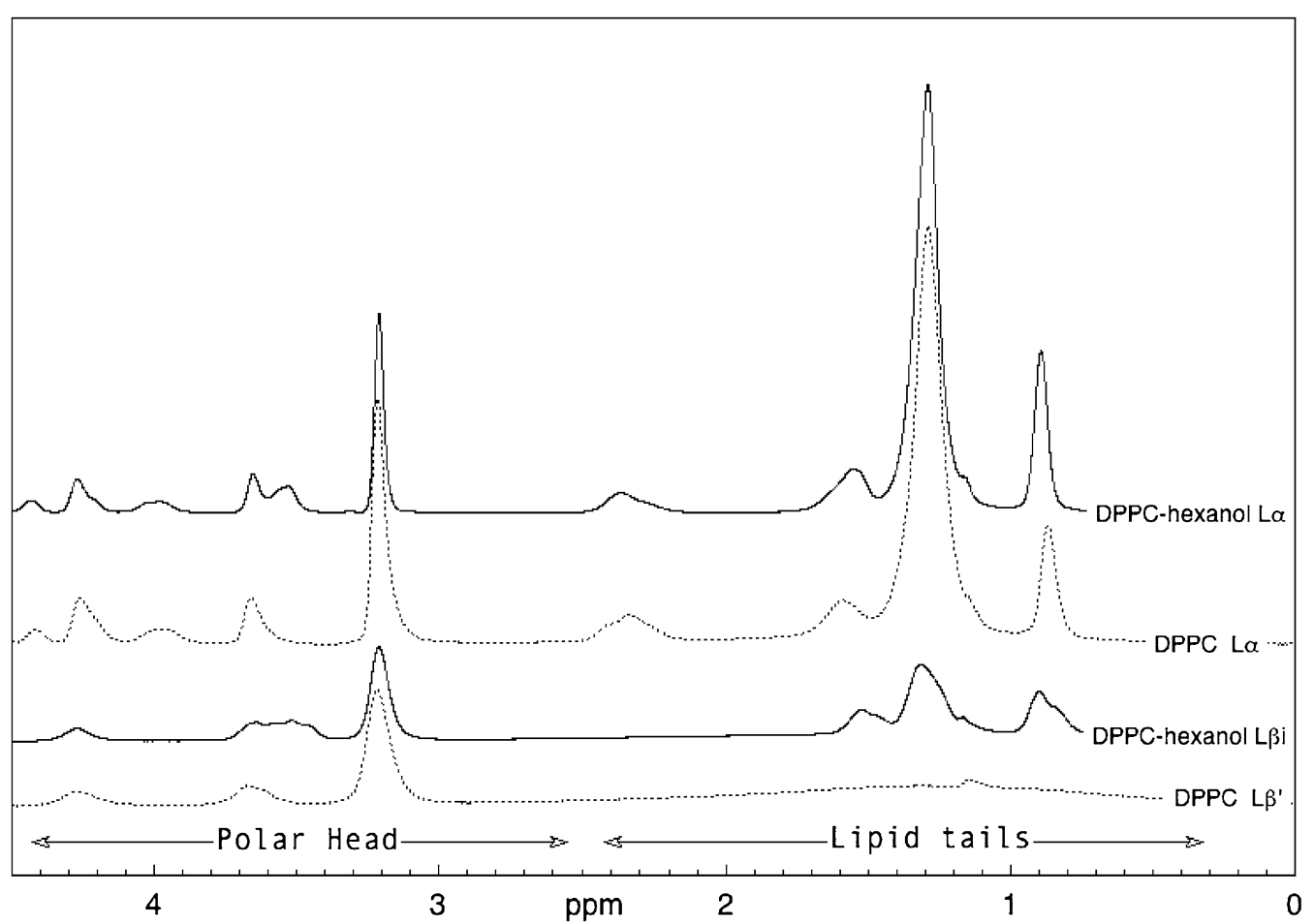

Fig. 2. $700 \mathrm{MHz}$ magic-angle spinning ${ }^{1} \mathrm{H}$ spectra for DPPC:hexanol: $\mathrm{D}_{2} \mathrm{O}$ [1:2:40] (continuous line) and DPPC: $\mathrm{D}_{2} \mathrm{O}$ [1:40] (dotted line) in the liquid and gel phase, respectively, at $5^{\circ}$ above and below the main transition temperature.

Table 1

Calculated quantities relative to DPPC and DPPC:hexanol multi bilayer structure

\begin{tabular}{lccccc}
\hline Sample & Volume $\left(\AA^{3}\right)$ & \% hydration by weight & Number of waters/lipid & $D / 2(\AA)$ & Head area $\left(\AA^{2}\right)$ \\
\hline DPPC & $1145 \pm 1$ & $18 \pm 1$ & $7.4 \pm 0.5$ & 30.01 & 45.5 \\
DPPC/hexanol & $1524 \pm 1$ & $13 \pm 1$ & $6.8 \pm 0.5$ & 28.98 & 59.6 \\
\hline
\end{tabular}

interdigitated phase for the DPPC:hexanol [1:2] membrane and confirm the insertion of alcohol with the polar portion close to phosholipid heads and with the chains in contact with the bilayer hydrophobic core. The structure interdigitated phase shows a greater disorder in the chains region then that observed in the ordinary gel phase.

\section{References}

[1] T.Adachi, H. Takahashi, K. Ohki and I. Hatta, Biophys. J. 68 (1995), 1850-1855.

[2] R.S. Cantor, Biochemistry 36 (1997), 2339-2344.

[3] R.S. Cantor, Biochemistry 42 (2003), 11891-11897.

[4] A.N. Dickey and R. Falle, Biophys. J. 92 (2007), 2366-2376.

[5] S.E. Feller, C.A. Brown, D.T. Nizza and K.Gawrisch, Biophys. J. 82 (2002), 1396-1404.

[6] A.A. Gurtovenko and J. Anwa, J. Phys. Chem. B 113 (2009), 1983-1992.

[7] M. Kranenburg and B. Smit, FEBS Letters 568 (2004), 15-18.

[8] R. Lipowsky and E. Sackmann, Structure and dynamics of membranes: from cells to vesicles, in: Handbook of Biological Physics, Vol. 1, Elsevier Science, Amsterdam, The Netherlands, 1995.

[9] L. Löbbecke and G. Cevc, Biochim. Biophys. Acta 1237 (1995), 59-69. 
[10] T.J. McIntosh, H. Lin, S. Li and C.-H. Huang, Biochim. Biophys. Acta 1510 (2001), 219-230.

[11] A. Mellier, Chem. Phys. Lipids 46 (1988), 51-56.

[12] J.F. Nagle and D.A. Wilkinson, Biophys. J. 23 (1978), 159-175.

[13] K. Ohki et al., Biochim. Biophys. Acta 1028 (1990), 215.

[14] L. Pauling, The Nature of the Chemical Bond, Cornell University Press, New York, 1960.

[15] U.R. Pedersen, G.H. Peters and P. Westh, Biophys. Chem. 125 (2007), 104-111.

[16] E.S. Rowe and J.M. Campion. Biophys. J. 67 (1994), 1888-1895.

[17] S.A. Simon and T.J. McIntosh, Biochim. Biophys. Acta 773 (1984), 169-172.

[18] J.L. Slater and C.-H. Huang, Prog. Lipid. Res. 27 (1988), 325-359.

[19] M. Suurkuusk and S.K. Singh, Chem. Phys. Lipids 94 (1998), 119-138.

[20] S. Tristram-Nagle, Y. Liu, J. Legleiter and J.F. Nagle. Biophys. J. 83 (2002), 3324-3335.

[21] S. Tristram-Nagle and J.F. Nagle, Chem. Phys. Lipids 127 (2004), 3-14.

[22] S. Tristam-Nagle, M.C. Wiener, C.P. Yang and J.F. Nagle, Biochemistry 26 (1987), 4288-4294.

[23] M.C. Wiener, R.M. Suter and J.F. Nagle, Biophys. J. 55 (1989), 315-325.

[24] M.C. Wiener, S. Tristram-Nagle, D.A. Wilkinson, L.E. Campbell and J.F. Nagle, Biochim. Biophys. Acta 938 (1998), $135-142$. 


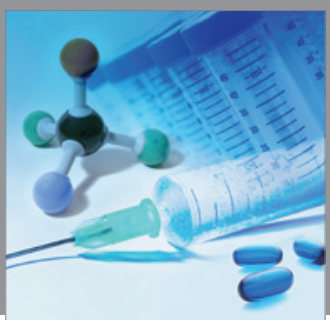

International Journal of

Medicinal Chemistry

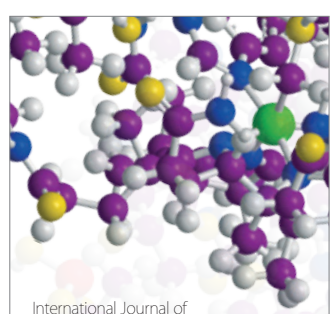

Carbohydrate Chemistry

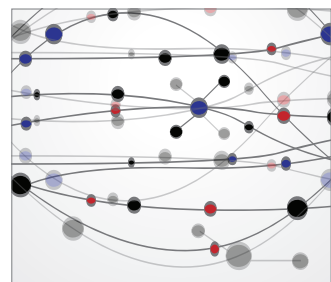

The Scientific World Journal
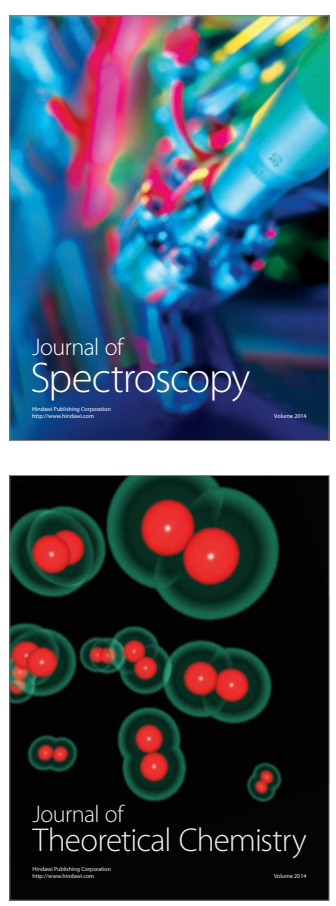
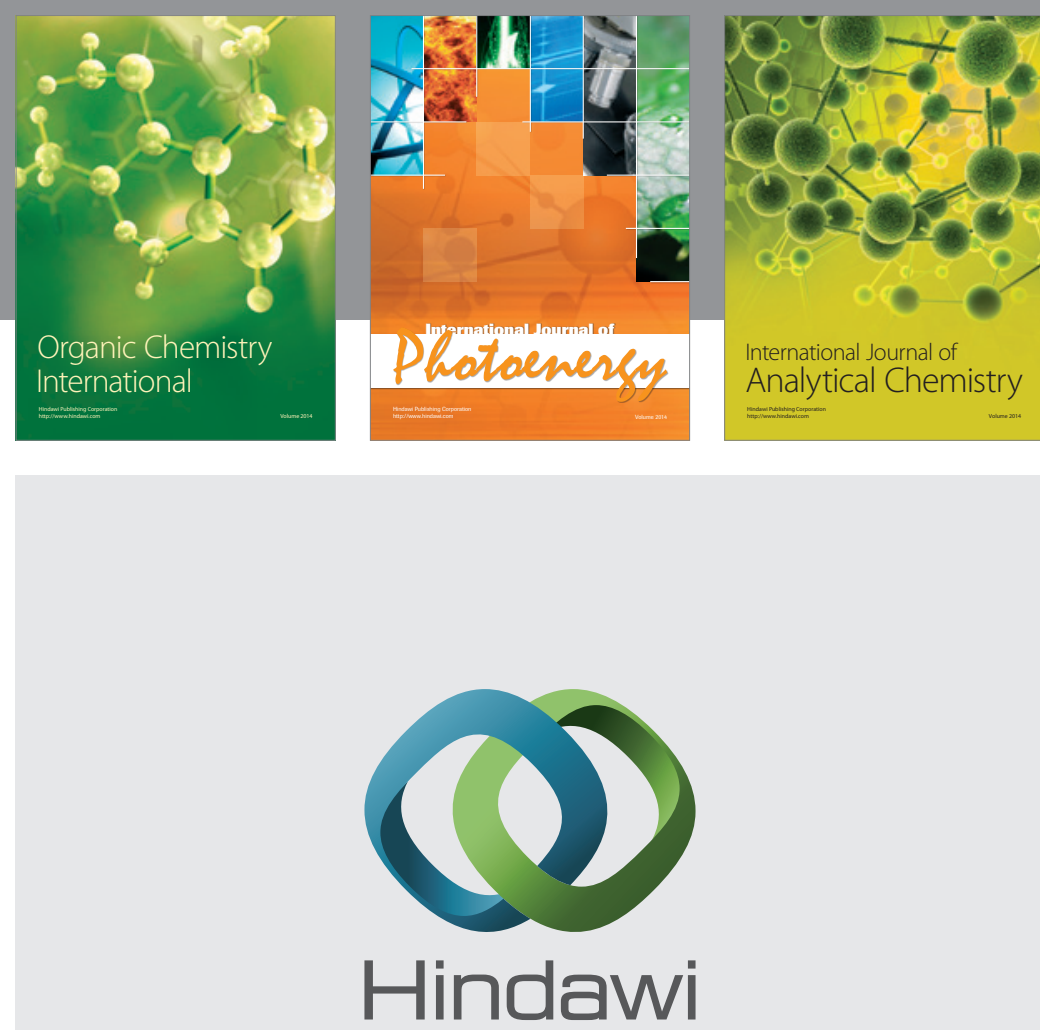

Submit your manuscripts at

http://www.hindawi.com
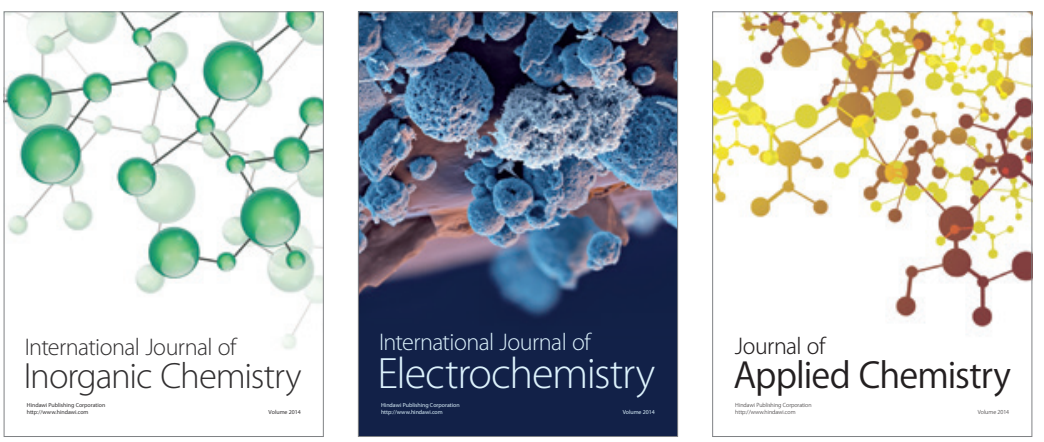

Journal of

Applied Chemistry
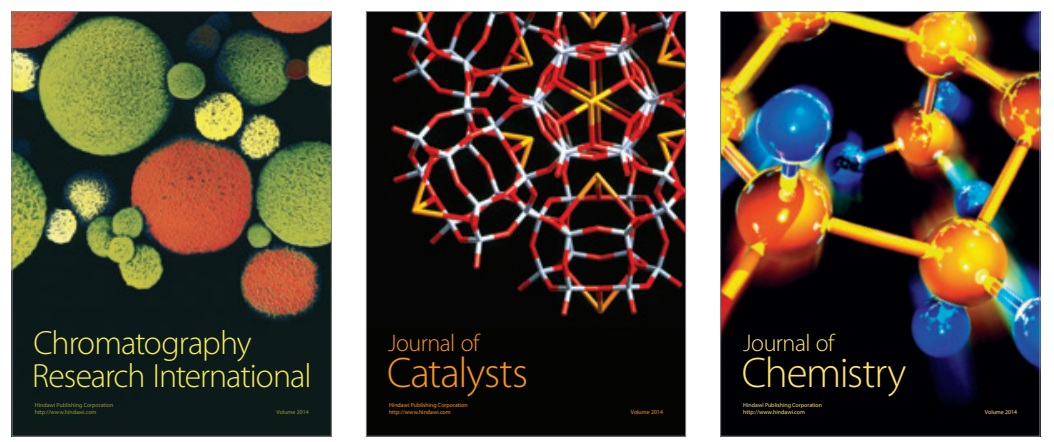
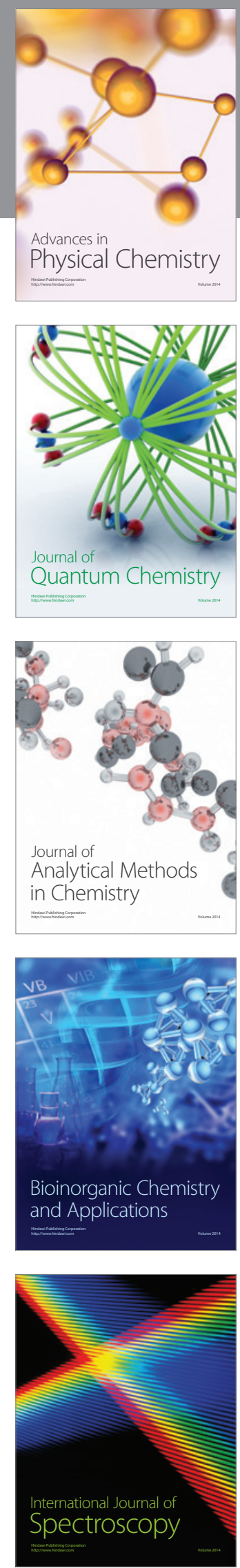